\title{
Controlled Rest in Position (CRIP): Consumer Perceptions in the United States
}

\author{
Scott R. Winter, Jason Carryl, and Stephen Rice \\ Florida Institute of Technology
}

\begin{abstract}
Controlled rest in position (CRIP) has been suggested as a viable countermeasure to the Federal Aviation Administration (FAA) in the ongoing efforts to combat in-flight pilot fatigue. It is already in use in other parts of the world; however, it is not currently permitted in the United States. While expert opinion suggested it poses little threat to flight safety, with valuable returns in terms of improved crew alertness and performance, there is some doubt about the level of consumer buy-in. This study completed a preliminary investigation into overall consumer willingness to fly, in the use of CRIP procedures in the United States. Two studies were completed to examine consumer perceptions toward the use of CRIP. It was found in both studies that male and female participants were less willing to fly when CRIP was used. Additionally, female participants were less willing than their male counterparts in both the control and experimental condition. In the second study, affect measures were collected and found to completely mediate the relationship between the use of CRIP and consumers' willingness to fly. Findings may provide both regulators and airlines data that could aid in their decision-making processes as it relates to implementing such practices in the United States.
\end{abstract}

\section{Introduction}

The issue of pilot fatigue in aviation has always been a widely discussed topic, and is of particular concern in today's work environment as airlines look to maximize revenue by working both man and machine to their limits. Controlled rest in position (CRIP) has been proposed as a solution to reduce pilot fatigue in flight, however not all regulatory bodies permit such procedures to be used, namely the Federal Aviation Administration (FAA). While there may be numerous factors that might have influenced why these procedures are prohibited in the United States, this study sought to look at the issue from the consumer perspective. What are their opinions on the matter; specifically, would they be willing to use air transportation with such protocols in effect? It was the aim of this study to provide such metrics to be able to provide to the aviation community at large, both ordinary airmen and regulators alike, information to help facilitate a global and united approach to combat pilot fatigue in aviation. The purpose of this study was to conduct an investigation into the level of consumer willingness to fly in the practice of CRIP in aviation. Consumer willingness to fly on a flight where CRIP protocols were in effect was studied in both a control and experimental group in the United States. This research investigated overall consumer perception, and explored the relationship gender has on the outcomes. 


\section{Literature Review}

Fatigue in commercial aviation proves an unceasing threat to flight safety, and viable countermeasures to combat the in-flight fatigue of pilots are a hotly debated topic. CRIP is, of course, one of the possible solutions, however there seems to be some disparity across the aviation community about its merits. Naturally, any new policies implemented by airlines not only have to meet regulatory guidelines on safety, but must also be accepted by the travelling public at large, who use the airlines. Since this study will be able to determine consumer 'buy-in', it may reduce airline uncertainty over adopting such a policy. This not only has the potential to save airlines time and money, but also by eliminating a major hurdle, could pave the way for a unified industry standard of either allowing or discontinuing the practice of CRIP to combat pilot fatigue in flight.

The issue of pilot fatigue has always been a major concern in the aviation industry, for operators and regulators alike. As aforementioned, the aim of this study is to seek consumer perception and willingness to fly in the countermeasures used to combat this issue, especially the solution of CRIP. In order to comprehensively approach this issue, consideration was given to the issues involved with pilot fatigue.

\section{Causes of Pilot Fatigue in Aviation}

It is widely upheld that pilot fatigue poses one of the greatest risks to flight safety, and there have been numerous studies done detailing the risk associated with having insufficient rest, as well as the factors that may lead to such a situation occurring. According to Hartzler (2013), one of the issues is extended duty periods associated especially with long haul international flights. Furthering that point, there is also sleep loss that is caused by unusually early or late duty periods. The issue of circadian disturbances as well as homeostatic factors also surfaced as contributing factors to pilot fatigue. Caldwell (2005) writes that long haul pilots who fly international routes attribute their fatigue to multiple time zone transitions, while the domestic, short haul pilots cite sleep deprivation and high workload as the major factors. Furthermore, Hartzler (2013) found environmental factors such as the level of automation employed in modern cockpits and pilots' roles as passive monitors, dim cockpit lighting, reduced blood oxygenation from operating at high altitudes, and pilot health to all contribute to overall fatigue.

\section{Hazards Associated with Pilot Fatigue}

These factors all lead to one of the dangerous manifestations of sleep deprivation; in flight micro-sleeps. According to Caldwell (2005), “...pilot micro-sleeps tended to occur most frequently during the cruise portion of long haul operations... and that micro-sleeps were more than 9 times more likely during nighttime flights compared to daytime flights. Of particular interest was the fact that such lapses apparently went unnoticed by the affected crewmembers" (p. 87). This finding serves to reinforce basic aeromedical knowledge, that pilots are susceptible to poor judgment because of fatigue and are often 
times unable to accurately assess his or her safety for flight.

\title{
Controlled Rest as a Fatigue Countermeasure
}

A theme in the empirical literature seems to indicate that in flight napping is beneficial to overall pilot alertness and thus, promotes safety. Hartzler (2013) writes, "Strategic naps...can help reverse performance deficits due to sleep loss or disruption, with naps as short as 10 min helping to reduce subjective sleepiness and improve neurophysical performance" (p. 312). It then comes as somewhat of a surprise that the Federal Aviation Administration (FAA) does not allow for napping for unaugmented flight crews in the United States, but a multiplicity of foreign carriers employ it as a fatigue mitigation tool. This policy of prohibiting CRIP seems to be at odds with findings of a NASA study conducted at the Ames facility in the 1990s. The NASA (1994) report found that between a control no-rest group and an experimental group that was allowed 40 minutes of sleep in flight, both objective measures such as response times, as well as subjective alertness ratings, proved superior for the rested group.

Perhaps the reluctance of the FAA to implement CRIP procedures is due to one of the side effects highlighted by the detractors: sleep inertia. It is defined in the work done by Hartzler (2013) as "...the period of cognitive and mood impairment, as well as hypovigilance, experienced immediately upon awakening...” (p. 314). Hartzler (2013) further adds that it was found that these detrimental effects can be observed approximately 30-60 minutes after waking up, and have even been observed up to 2 hours after awakening. For as ominous as that may appear, there are several simple industry mitigation solutions to sleep inertia, such as limiting rest periods to cruise flight, and requiring the pilots to be awake for a prescribed amount of time before commencing descent and arrival procedures. Caldwell (2005) suggests:

As long as technological and economic factors strain available personnel resources and basic human capabilities, fatigue will continue to be an aviation risk factor. However, systematic fatigue education... the implementation of scientifically based scheduling practices...will optimize the safety and well-being of crews, passengers, and payloads everywhere. (p. 194)

\begin{abstract}
Affect
As was previously mentioned, there exists a myriad of factors that may influence consumer willingness, and internal biases and opinions may influence consumer behavior. Affect is one particular area where research on evaluative processes has been increasing (Bodenhausen, 1993; Bower, 1991; Clore, Schwarz, \& Conway, 1994; Forgas, 1995; Loewenstein, 1996; Schwarz \& Clore, 1996; Zajonc, 1998). More specifically, this research has investigated the role of emotional influence on human decision-making. Some studies suggest that emotions are used when information needs to be processed quickly (Frijda, 1986; Levenson, 1994; Oatley \& Johnson-Laird, 1996) and when longer-term,
\end{abstract}


more deliberate decisions must be made (Johnson-Laird \& Oatley, 1992; Lazarus, 1991; Schwarz, 1990; Simon, 1967; Tooby \& Cosmides, 1990).

While previous research (Trafimow \& Sheeran, 1998, 2004; Trafimow et al., 2004) has shown that affect and cognition are separate entities, research on evaluative judgments has demonstrated that emotions do have a role in the decision-making process (Clore, Schwarz, \& Conway, 1994; Schwarz, 1990; Schwarz \& Clore, 1983, 1988, 1996). It is possible when individuals are presented with new information, they may respond emotionally or at least have their response influenced by emotions.

Dholakia (2001) brings up the issues of perceived risk and product involvement as affective factors that may influence customer willingness. The author found that risk perceptions are a function of cognitive evaluation of a product by a consumer, which may cause airlines and regulators alike some unease, as travelers are all unique and have personalized thought processes. Dholakia (2001) goes on to state that there was some evidence to support that perceived risk affects consumers' product involvement. Product involvement is defined as "an internal state variable that indicates the amount of arousal, interest or drive evoked by a product class" (Dholakia, 2001, p. 1341). This would then suggest that as has already been happening, the safer a product class is perceived to be, the higher the likelihood of it being utilized. In terms of CRIP, it has been largely supported by the experts, as well as been successfully implemented overseas, so that begs the question why the lag in the U.S. policymaking?

\section{Willingness as a Metric}

As previously mentioned, the focus of this paper is to investigate consumer perceptions, in terms of the level of consumer willingness to fly in CRIP protocols. Writing in thefreedictionary (2014) defines willingness as "done, given, accepted, or borne voluntarily or ungrudgingly". By using willingness as a metric, consumer views toward controlled rest procedures can now be quantified. Key to any new technology or policy being introduced is consumer acceptance or buy in. Work by Pramatari and Theotokis (2009) identifies personality characteristics, service and technology characteristics, and situational factors as items that can affect consumer perceptions. Naturally, even the best analyses and safeguards that the airlines implement cannot guarantee positive consumer perceptions, nor can they intrinsically alter individual personalities to support CRIP. What they can do, as evidenced in the work by Pramatari and Theotokis (2009), is extol the virtues of the system in terms of ease of use and complexity, or lack thereof. That being said, work by Zhao and Kling (2004) might once again bring pause to the regulators and top airline management alike. According to Zhao and Kling (2004):

How much one is willing to pay (or accept) for a good at a particular point in time will depend on...the ability to reduce the risk of a bad purchase or sale by gathering more information, and the ease of later reversing the transaction. (p. 504) 
One of the unique characteristics of the airline product is that there is little to no way of evaluating the product beforehand, as there are so many variables that could potentially affect product quality. For example, there could easily be delays to on-time service as a result of weather or maintenance issues. Furthermore, reversing the purchase is usually quite difficult if not impossible. In this study the researchers are specifically evaluating willingness to fly. If the customer has any reservations about CRIP procedures, there could well be a reduction in airline passenger traffic, which would naturally concern management. This study may provide useful information on consumer perceptions toward the use of CRIP procedures.

The scale used in this study was a valid and reliable instrument to measure consumer's willingness to fly. Rice et al. (accepted) completed the scale development and validation. Consumers were used in all stages of the instrument creation. This included stages of word generation, word pairing, validation, discrimination, and scenario-based applications. The final instrument consisted of 7 statements to measure a consumer's willingness to fly using a 5-point Likert scale from Strongly Disagree to Strongly Agree.

\section{Study 1 Research Questions and Hypotheses}

Study 1 sought to answer the following research questions:

1) What is the overall willingness to fly of consumers when controlled rest procedures are in effect?

2) How does gender affect consumer willingness to fly ratings when controlled rest in position is used?

The following Hypotheses were posited for Study 1:

$\mathrm{H}_{1}$ - Based on the literature, it was predicted that there would be a decrease in willingness to fly ratings when controlled rest in position was used.

$\mathrm{H}_{2}$ - It was predicted that there would be some difference in willingness to fly ratings between the genders if controlled rest in position is used.

$\mathrm{H}_{3}$ - There would be possible interactions between the variables.

\section{Study 1 Methodology}

\section{Participants}

Two hundred and fifty-four total participants for this study were sampled from the American population, 159 males $(M=32.91$ years, $S D=9.91)$ and 95 females $(M=34.83$ years, $S D=12.62$ ) and were limited to persons aged 18 years or older. They were sourced from the online population of persons who complete electronic surveys for compensation via Amazon's ® Mechanical Turk @ (MTurk). This is an interface available through Amazon that provided electronic means to facilitate the compensation of participants for 
the completion of tasks. Participants received a small monetary compensation for their time. Research has shown that MTurk provides data that is as reliable as laboratory data (Buhrmester, Kwang, \& Gosling, 2011; Germine, et al., 2012).

\section{Procedure and Materials}

The survey was distributed electronically via FluidSurveys $₫$ on Amazon's ® Mechanical Turk ®. Participants were asked questions about their willingness to fly rating if controlled rest in position procedures are being practiced. In the control condition participants read the following scenario: Imagine that you are on an overnight 7-hour international flight between two major cities with two pilots on-board. You learn that the airline requires both pilots to remain awake for the duration of the flight. The scenario in the experimental condition read: Imagine that you are on an overnight 7-hour international flight between two major cities with two pilots on-board. You learn that the airline allows that one pilot at a time may nap in the cockpit provided the other remains awake and a flight attendant has been notified that one of the pilots will be napping. The flight attendant serves as a backup to ensure the nap only lasts for the specified duration.

Responses measuring willingness used a Likert-type scale from strongly disagree (-2), to strongly agree $(+2)$, with a neutral option included, and were analyzed to quantify consumer results. This scale was a valid and reliable instrument that has been shown to measure consumer's willingness to fly (Rice et al., accepted). Demographic information was also collected and analyzed to determine if there were any gender differences, and then participants were debriefed on the study.

\section{Study 1 Results}

A valid and reliable instrument was used in the study to test the dependent variable of willingness to fly (Rice et al., accepted); however, to verify internal consistency, a Cronbach's Alpha test was conducted. Scores ranged from .96 to .98 , which implied high consistency between answers, allowing the data to be averaged into a single score for each participant.

A 2 × 2 Factorial ANOVA analysis was conducted on the data using condition and gender as between participant factors. The main effect of condition was found to be significant, $F(1,250)=20.16, p<0.001, \eta p^{2}=0.075$, as well as that of gender, $F(1,250)$

$=7.31, p=0.007, \eta p^{2}=0.03$. There was no significant interaction between gender and condition, $F(1,250)=0.045, p=0.83, \eta p^{2}=0.00$. Study results are depicted in Figure 1 . 


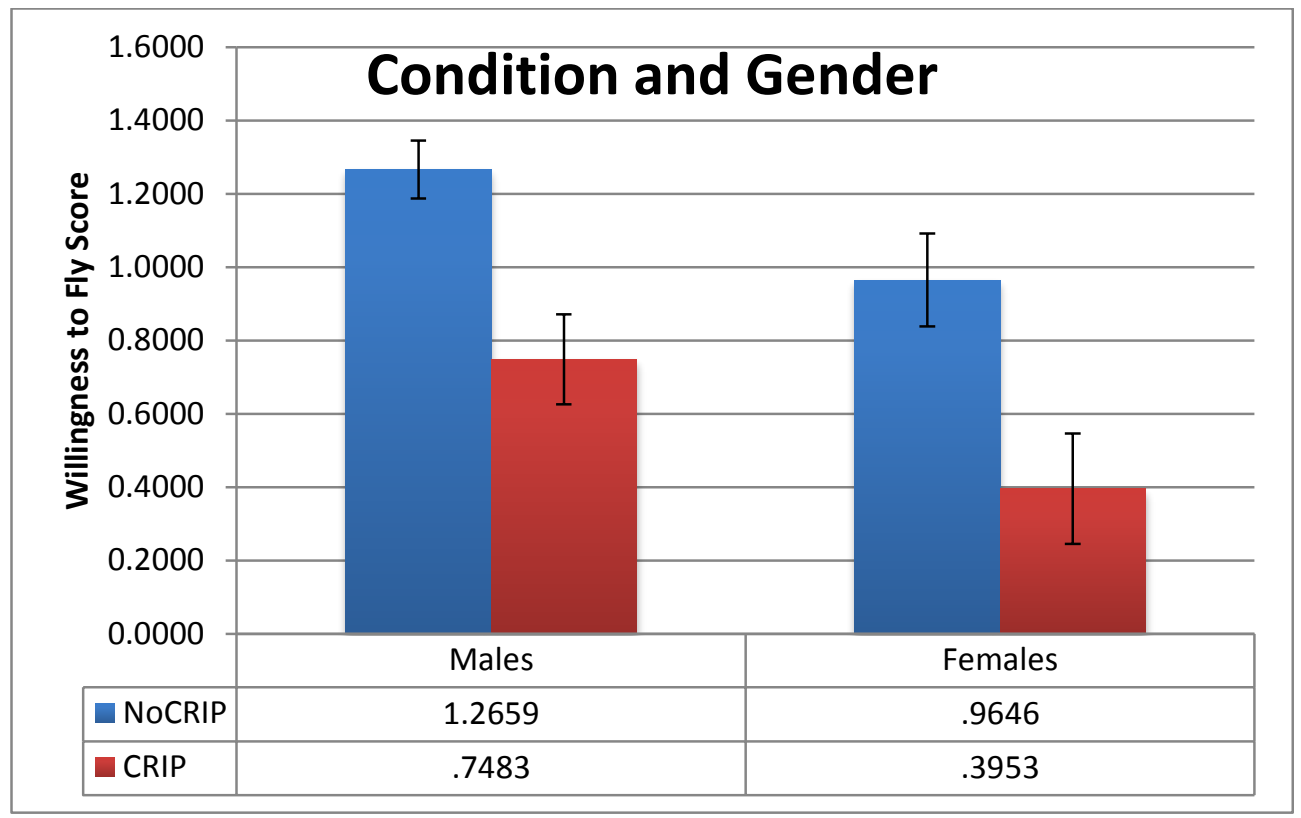

Figure 1. Study 1 Willingness to Fly Data. SE bars included.

\section{Study 2 Research Questions and Hypotheses}

The second study sought to replicate the findings of Study 1. Therefore the research questions and hypotheses remained the same. Additionally, the researchers wanted to determine if Affect would mediate, at least partially, the relationship between the condition and willingness to fly score. Based on similar studies (Remy, Winter, \& Rice, 2014; Winter, Rice, \& Mehta, 2014), it was hypothesized that Affect would mediate, at least partially, the relationship between the condition and willingness to fly.

\section{Study 2 Methodology}

\section{Participants}

Two hundred and seventy-six participants were sampled from the American population using Amazon's ${ }^{\circledR}$ MTurk ${ }^{\circledR}$. Participants included 156 males $(M=31.22, S D=8.51)$ and 142 females $(M=32.95, S D=10.27)$ that were all over the age of 18 from the United States.

\section{Procedure and Materials}

The procedure for Study 2 was identical to Study 1, except participants in Study 2 were additionally asked 3 questions related to Affect (Remy, Winter, \& Rice, 2014; Winter, Rice, \& Mehta, 2014) and asked to indicate how the scenario made them feel on a 7-point Likert scale from extremely bad/negative/unfavorable (-3) to extremely 
good/positive/favorable $(+3)$, with a neutral option of 0 before being asked to rate their willingness to fly.

\section{Study 2 Results}

Study 2 used the same valid and reliable instrument from Study 1. The two dependent variables (willingness to fly and Affect) were subjected to a Cronbach's Alpha analysis for internal consistency. The values for willingness to fly ranged from .96 to .98 and for Affect both scores were .97; therefore, due to the high internal consistency for each of the dependent variables a single average score was produced for willingness to fly and Affect for each participant.

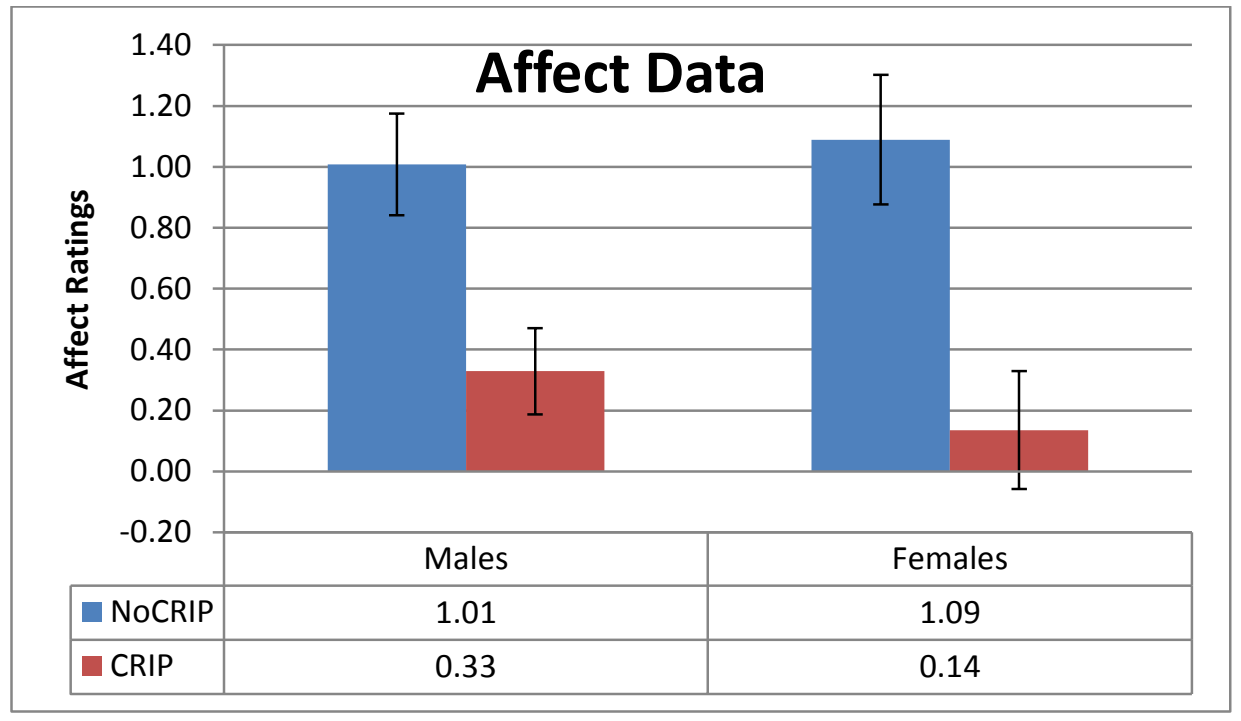

Figure 2. Study 2 Affect Data. SE bars included.

\section{Factorial Analyses}

A $2 \times 2$ Factorial ANOVA analysis was conducted on the data using condition and gender as between participant factors. For the Affect data, the main effect of condition was found to be significant, $F(1,272)=45.09, p<0.001, \eta p^{2}=0.072$. The main effect of gender was found to not be significant, $F(1,272)=0.10, p>0.05, \eta p^{2}=0.00$ nor was the interaction, $F(1,272)=0.597, p>0.05, \eta p^{2}=0.00$. Figure 2 depicts the Affect data. For the willingness to fly data, the main effect of condition was found to be significant, $F(1$, $272)=15.04, p<0.001, \eta p^{2}=0.052$, as well as that of gender, $F(1,272)=6.40, p<0.05$, $\eta p^{2}=0.023$. There was no significant interaction between gender and condition, $F(1,272)$ $=0.047, p=0.83, \eta p^{2}=0.00$. Willingness to fly data are depicted in Figure 3 . 


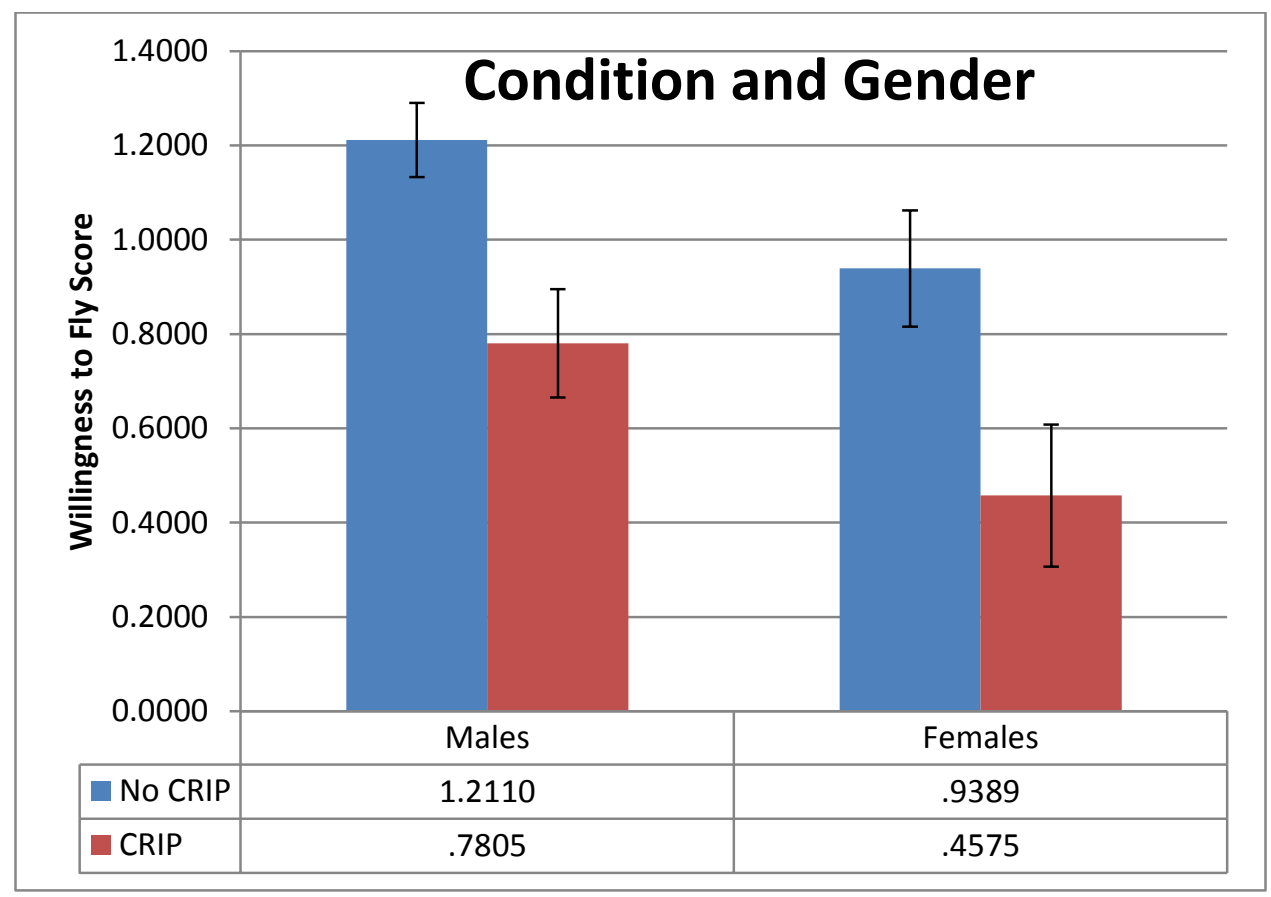

Figure 3. Study 2 Willingness to Fly Data. SE bars included.

\section{Mediation Analysis}

The mediation analysis for males is presented in Figure 4. In order to conduct the mediation analysis, the correlation between condition and willingness to fly was first found to be significant, $r=.242, p<0.05$, showing that the initial variable correlated with the outcome variable. The standardized path coefficients were: condition to affect $(.242, p<$ $0.05)$; affect to willingness to fly $(.614, p<0.001)$; condition to willingness to fly controlling for affect $(-.093, p=.147)$. These data show that Affect has complete mediation on the relationship between use of controlled rest and willingness to fly for males.

The mediation analysis for females is presented in Figure 5. In order to conduct the mediation analysis, the correlation between condition and willingness to fly was first found to be significant, $r=.218, p<0.05$, showing that the initial variable correlated with the outcome variable. The standardized path coefficients were: condition to affect $(.293, p<$ $0.05)$; affect to willingness to fly $(.826, p<0.001)$; condition to willingness to fly controlling for affect $(-.023, p=.675)$. These data show that Affect has complete mediation on the relationship between use of controlled rest and willingness to fly for females. 


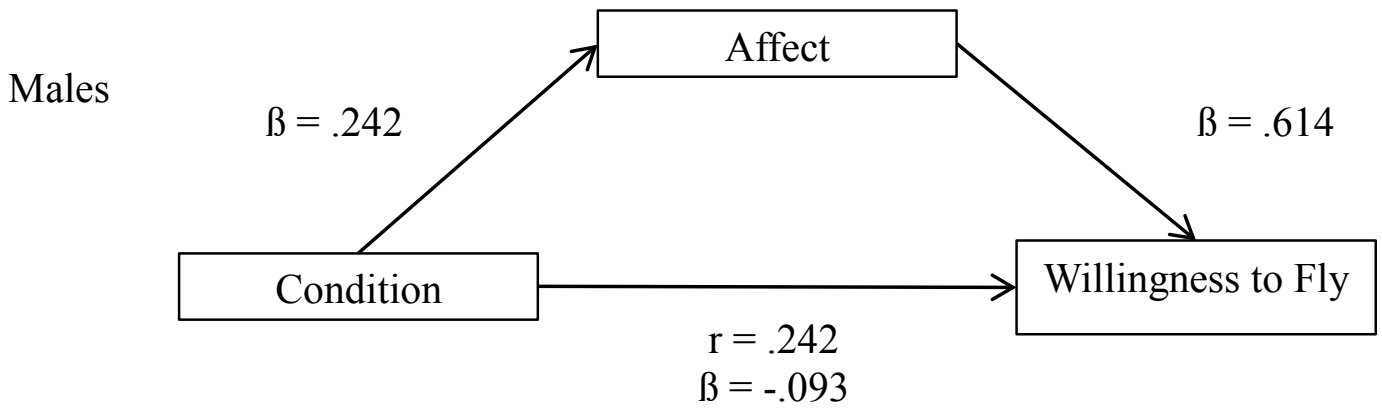

Figure 4. Path analysis for Males.

Females

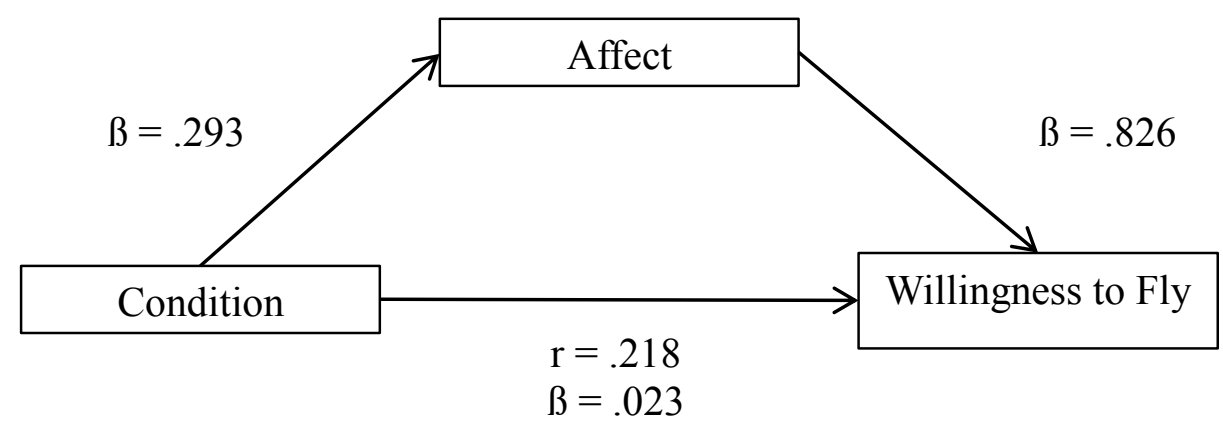

Figure 5. Path analysis for Females.

\section{General Discussion}

The purpose of this paper was to investigate both the general public perception of CRIP, as well as to examine any differences in responses between the genders. Using an instrument on consumer's willingness to fly, the study examined for differences based on the use of controlled rest procedures, gender, and if Affect mediated any relationship between condition and gender.

In the first hypothesis, data indicated a significant difference in willingness to fly ratings between the controlled rest usage scenario and no controlled rest conditions. Despite expert opinion on the benefits of controlled rest, participants were much more willing to fly when controlled rest was not practiced. This finding seems to suggest unfamiliarity on the part of the participants with the concept of controlled rest, and offers regulators an idea of likely public feedback if such rules were implemented (Pramatari \&Theotokis, 2009). While generally the responses did not indicate an unwillingness to fly while controlled rest in position was being used (average responses still above 0), public education is likely necessary to convince the general population of the benefits of using such procedures. One would imagine if the public was made aware of the role of automation, coupled with the opportunity to rest in the low workload cruise portion of flight and be more alert for the high workloads of approach to landing, their opinions may change somewhat. 
Similarly, the null hypothesis was also rejected in the second hypothesis, as there was a significant difference in willingness to fly ratings between the genders. The data indicated males were more willing than females when it came to flying while controlled rest was being used. This somewhat contradicts findings by Porter, Donthu, and Baker (2012), which stated there typically is no significant difference in levels of willingness to fly between the genders. This finding encourages further research into determining why this might be true. Perhaps answers might be found when comparing how the genders define and assess risk, which according to these findings, suggests a more risk averse nature of females.

Finally, it was predicted that Affect would at least partially mediate the relationship between the condition, use of controlled rest or not, and willingness to fly score. For both males and females, Affect was found to completely mediate this relationship. While research has demonstrated the advantages of strategic use of napping, it is plausible to understand a passenger's potential shock to learn of a pilot napping in the aircraft. As reported by Dholakia (2001), when consumers perceive a situation as being more risky than an alternative, it is possible that they may respond emotionally. For countries that have implemented controlled rest, very specific procedures and policies are in place to ensure that the practice is completed safely. With Affect providing mediation, it is possible that providing education to consumers of the benefits of CRIP may help them understand the value of this procedure and benefits it can provide to help keeping the flight crew alert during critical phases of flight.

\section{Practical Implications}

The results of this study may be of practical value to both airline management and industry regulators alike. In the cases of both parties, it is of critical importance to determine consumer buy-in when new rules or procedures are proposed, as there is a strong risk aversion to isolating any portion of the customer base. These early findings help to paint a picture of general consumer perceptions on the implementation of controlled rest, and so allow both invested parties to make more informed decisions. Based on study findings, it seems that a public education program will need to be implemented well in advance of implementing controlled rest regulations, since both genders were significantly less willing to fly when such procedures were used. One would want to ensure the best possible opportunity for a cognitive, rather than emotional assessment from the public with regard to their support of these procedures.

\section{Limitations and Recommendations for Further Research}

Naturally, the findings presented in this study must be considered with the study's limitations. The major limitation of this study is the fact that the population surveyed was only persons who completed online human intelligence tests, thereby limiting the generalizations to that particular subset, which may or may not be representative of the larger population. Additionally, the participants may not accurately represent the travelling 
public that uses commercial aviation, as there was no requirement to be a user of commercial air service to participate in the study. Opportunities for further study are numerous. Questions were raised in the discussion about whether the overall consumer perception ratings were influenced by an emotional response rather than a cognitive assessment. This might be investigated by modifying the study to compare responses between participants who received a factual briefing on controlled rest procedures and those who did not. The addition of affective measures may also assist in confirming that research question. Additionally, more research could be conducted to determine why there was a significant difference in willingness to fly ratings between the genders, which is a quasi-experimental variable.

\section{Conclusions}

This study examined the consumer perceptions of the American population in terms of their willingness to fly rating and the use of CRIP procedures on commercial aviation flights. Results indicated a lower average consumer willingness to fly rating while these procedures were in effect compared to when they were not, and that between the genders, females were less willing than males for the same condition. These findings can be used by airlines operating in United States as well as the Federal Aviation Administration to garner insight into consumer buy in, and can hopefully guide policy-making to determine the most effective countermeasure to combat in flight pilot fatigue. 


\section{References}

Bodenhausen, G. V. (1993). Emotions, arousal, and stereotypic judgments: A heuristic model of affect and stereotyping. In D .M. Mackie \& D. L. Hamilton (Eds.), Affect, cognition, and stereotyping: Interactive processes in group perception (pp. 13-37). San Diego, CA: Academic Press.

Bower, G. H. (1991). Mood congruity of social judgment. In J. Forgas (Ed.), Emotion and social judgment (pp. 31-54). Oxford, UK: Pergamon.

Buhrmester, M., Kwang, T., \& Gosling, S. D. (2011). Amazon's Mechanical Turk: A new source of inexpensive, yet high-quality data? Perspectives on Psychological Science, 6(3), 3-5.

Caldwell, J. A. (2005). Fatigue in aviation. Travel Medicine and Infectious Disease, 3(2), 85-96. doi:http://dx.doi.org/10.1016/j.tmaid.2004.07.008

Clore, G. L., Schwarz, N., \& Conway, M. (1994). Cognitive causes and consequences of emotion. In R. S. Wyer, Jr. \& T. K. Srull (Eds.), Handbook of social cognition (2nd ed., pp. 323-417). Hillsdale, NJ: Erlbaum.

Dholakia, U. M. (2001). A motivational process model of product involvement and consumer risk perception. European Journal of Marketing, 35(11), 1340-1360.

Frijda, N. H. (1986). The emotions. Cambridge, UK: Cambridge University Press. Bulletin, 117, 39-66.

Forgas, J. P. (1995). Mood and judgment: The affect infusion model (AIM). Psychological Bulletin, 117, 39-66.

Germine, L., Nakayama, K., Duchaine, B.C., Chabris, C.F., Chatterjee, G., \& Wilmer, J.B. (2012) Is the web as good as the lab? Comparable performance from web and lab in cognitive/perceptual experiments. Psychonomic Bulletin \& Review, 19(5), 847857.

Hartzler, B. M. (2013). Fatigue on the flight deck: The consequences of sleep loss and the benefits of napping. Accident Analysis and Prevention, 62, 309-318. doi:http://dx.doi.org/10.1016/j.aap.2013.10.010

Lazarus, R.S. (1991). Emotion and adaptation. New York: Oxford University Press.

Levenson, R. (1994). Human emotion: a functional view. In P. Ekman \& R. J. Davidson (Eds.), The nature of emotion (pp. 123-126). New York: Oxford University Press. 
Loewenstein, G. (1996). Out of control: Visceral influences on behavior. Organizational Behavior and Human Decision Processes, 65, 272-292.

NASA (1994). Crew Factors in Flight Operations IX: Effects of Planned Cockpit Rest on Crew Performance and Alertness in Long-Haul Operations. (NASA Technical Memorandum 108839). Moffett Field, California: Ames Research Center.

Oatley, K., \& Johnson-Laird, P. N. (1996). The communicative theory of emotions: Empirical tests, mental models, and implications for social interaction. In L. L. Martin \& A. Tesser (Eds.), Striving and feeling: Interactions among goals, affect, and self-regulation. Hillsdale, NJ: Erlbaum.

Porter, C. E., Donthu, N., \& Baker, A. (2012). Gender differences in trust formation in virtual communities. Journal of Marketing Theory and Practice, 20(1), 39-58.

Pramatari, K., \& Theotokis, A. (2009). Consumer acceptance of RFID-enabled services: A model of multiple attitudes, system characteristics and individual traits. Rochester: Social Science Research Network. doi:http://dx.doi.org/10.2139/ssrn.1329907

Remy, B., Winter, S. R., \& Rice, S. (2014, April). American aviation consumer's trust in pilots. Presentation at the $7^{\text {th }}$ annual Human Factors and Applied Psychology Student Conference, Daytona Beach, FL.

Rice, S., Mehta, R., Dunbar, V., Oyman, K., Ghosal, S., Oni, M.D. \& Oni, M.A. (accepted). A valid and reliable scale for consumer willingness to fly. Proceedings of the 2015 Aviation, Aeronautics, and Aerospace International Research Conference, Phoenix, AZ.

Schwarz, N. (1990). Feelings as information: Informational and motivational functions of affective states. In E. T. Higgins \& R. M. Sorrentino (Eds.), Handbook of motivation and cognition: Foundation s of social behavior (Vol. 2, pp. 527-561). New York: Guilford Press.

Schwarz, N. \& Clore, G.L. (1983). Mood, misattribution and judgments of well-being: Informative and directive functions of affective states. Journal of Personality and Social Psychology, 45, 513- 523.

Schwarz, N. \& Clore, G. (1988). How do I feel about it? The information function of affective states. In K. Fiedler \& J.P. Forgas (Eds.), Affect, cognition, and social behavior: New evidence and integrative attempts (pp. 44-63). Toronto: C.J. Hogrefe.

Schwarz, N., \& Clore, G. (1996). Feelings and phenomenal experiences. In E. T. Higgins \& A.W. Kruglanski (Eds.), Social psychology: Handbook of basic principles (pp. 
433-465). New York: Guilford Press.

Simon, H. A. (1967). Motivational and emotional controls of cognition. Psychological Review, 74, 29-39.

Thefreedictionary (2014). Retrieved from: http://www.thefreedictionary.com/willingness

Tooby, J., \& Cosmides, L. (1990). The past explains the present: Emotional adaptations and the structure of ancestral environments. Ethology and Sociobiology, 11, 375424.

Trafimow, D., \& Sheeran, P. (1998). Some tests of the distinction between cognitive and affective beliefs. Journal of Experimental Social Psychology, 34, 378-397.

Trafimow, D., \& Sheeran, P. (2004). A theory about the translation of cognition into affect and behavior. In G. Maio \& G. Haddock (Eds.), Contemporary perspectives in the psychology of attitudes: The Cardiff Symposium (pp. 57-76). London: Psychology Press.

Trafimow, D., Sheeran, P., Lombardo, B., Finlay, K. A., Brown, J., \& Armitage, C.J. (2004). Affective and cognitive control of persons and behaviors. British Journal of Social Psychology, 43, 207-224.

Winter, S. R., Rice, S., \& Mehta, R. (2014). Aviation consumers' trust in pilots: A cognitive or emotional function. International Journal of Aviation, Aeronautics, and Aerospace, 1(1), 1-18.

Zajonc, R. (1998). Emotions. In D. Gilbert, S. Fiske, \& G. Lindzey (Eds.), The handbook of social psychology (Vol. 1, pp. 591-632). New York: Oxford University Press.

Zhao, J., \& Kling, C. L. (2004). Willingness to pay, Compensating variation, and the cost of commitment. Economic Inquiry, 42(3), 503-517. Retrieved from: http://search.proquest.com/docview/200862760?accountid=27313 